\title{
Dexmedetomidine for every Cesarean delivery...maybe not?
}

\author{
Lesley Bautista, MD, FRCPC · Ronald B. George, MD, FRCPC
}

Received: 18 February 2019/Accepted: 18 February 2019/Published online: 27 March 2019

(C) Canadian Anesthesiologists' Society 2019

Perioperative shivering is a commonly observed clinical problem well known to anesthesiologists caring for women undergoing a Cesarean delivery (CD). The median incidence of shivering in parturients having labour epidural analgesia or CD with epidural or spinal anesthesia has been estimated to be $52 \%{ }^{1}$ Severe shivering causes physiologic stress, interferes with patient monitors, and decreases the comfort and overall satisfaction of women during childbirth. ${ }^{2}$

Many studies have evaluated pharmacologic agents to treat shivering associated with neuraxial anesthesia in both parturients and the general population. Previously studied agents including butorphanol, ${ }^{3} \quad$ clonidine, $^{4}$ fentanyl, ${ }^{5}$ meperidine, ${ }^{6}$ tramadol, ${ }^{7}$ and dexmedetomidine ${ }^{8}$ decrease established shivering following neuraxial anesthesia. Nevertheless, side effects such as hypotension, nausea, and vomiting, as well as excessive sedation limit the utility of some agents in certain patient populations.

Dexmedetomidine, a selective $\alpha-2$ agonist, was introduced in clinical practice in the United States in 1999. It is approved by Health Canada as a short-term sedative for mechanically ventilated adult patients in the intensive care unit and sedation of non-intubated patients prior to and/or during surgical procedures. It has since been approved and is widely used in pediatric populations and for procedural sedation of non-intubated patients. ${ }^{9}$ Nevertheless, the clinical use of dexmedetomidine has expanded to various off-label uses. In obstetrical anesthesia, it has been used as part of multi-modal

L. Bautista, MD, FRCPC $(\bowtie) \cdot$ R. B. George, MD, FRCPC

Department of Women's \& Obstetric Anesthesia, IWK Health Centre, Dalhousie University, 5850/5980 University Avenue, P.O. Box 9700, Halifax, NS, Canada

e-mail: 1.bautista@mun.ca intravenous labour and post-CD analgesia, ${ }^{10-13}$ as a neuraxial adjunct in the epidural space for labour, ${ }^{14}$ and intrathecally for shivering prevention during CD. ${ }^{15}$

In this issue of the Journal, Lamontagne et al. ${ }^{2}$ present a novel use of this drug in parturients to treat shivering during CD. The authors conducted a singlecentre, randomized, double-blind, placebo-controlled, parallel-group study in 80 healthy parturients undergoing CD with neuraxial anesthesia (spinal or epidural) who showed significant shivering after delivery. Participants were randomized to receive either a $30 \mu \mathrm{g}$ dexmedetomidine $i v$ bolus or an equal volume of normal saline. The authors found that 15 minutes after administration, the shivering had completely stopped in $90 \%$ of the intervention group vs only $23 \%$ of the control group (relative risk of not shivering, 4.0; 95\% confidence interval, 2.2 to 7.2). Significant bradycardia, a commonly observed adverse effect of intravenous dexmedetomidine, was not observed in either group, and hypotension was present in just one intervention group patient. This led the authors to conclude that shivering during CD could be treated effectively and safely with dexmedetomidine.

This study provides an intriguing and possibly compelling new option for treatment of a common problem in the obstetrical population. When severe, shivering is a nuisance to the anesthesiologist monitoring a patient, and impacts the woman's experience and satisfaction with our care. Based on this study, are we ready to routinely use dexmedetomidine to treat shivering in obstetrical patients given this is an offlabel (and possibly not cost effective) option? Before we convince our institutions to allow dexmedetomidine to be used for this consideration, it is important to consider the study's limitations. 
Lamontagne et $a .^{2}$ included labouring and nonlabouring parturients that required $\mathrm{CD}$. The physiology of parturients changes significantly when in labour. Labouring parturients have been reported to have both thermoregulatory and non-thermoregulatory shivering, with and without epidural analgesia. ${ }^{16}$ Despite normal increases in temperature during labour ${ }^{17}$ and with epidural analgesia, ${ }^{18}$ shivering can still occur and its mechanism may be different from thermoregulatory shivering. ${ }^{1}$ In addition, labouring patients are often exposed to epidural analgesia and its effects on thermoregulation and shivering for hours prior to $\mathrm{CD}$. Therefore, a labouring parturient with epidural analgesia and a non-labouring parturient with spinal anesthetic for $\mathrm{CD}$ are conceivably two very different patient populations.

Additionally, and as a result of their inclusion criteria, each group contained some parturients who received spinal anesthesia (70\%) and some who received epidural anesthesia (30\%) for CD. Though both spinal and epidural anesthesia decrease the shivering threshold, it is unclear whether they affect the shivering threshold in different ways. ${ }^{1}$ While spinal anesthesia results in a dense block below a certain dermatome, epidural often spares the sacral dermatomes, which may result in decreased block of thermal input in epidural $v s$ spinal anesthesia at a given block height. ${ }^{1}$ Spinal anesthesia rapidly induces hypothermia and a lower core temperature, while core temperatures in epidural anesthesia decrease slowly and linearly. ${ }^{19}$ Both techniques decrease core temperature at the same rate beyond 30 min. ${ }^{19}$ Despite typically more dramatic physiologic changes and a lower core temperature, shivering with spinal anesthesia has been observed to be less intense than with epidural anesthesia. ${ }^{19}$

The authors argue that using a broad obstetrical population improved the generalizability of their study. The authors still managed to have comparable intervention and control groups with regards to active labour and identical numbers of patients with epidural or spinal anesthesia, even though they did not stratify for these factors. This issue of subgroups with possible discordant or additive outcomes gives us uncertain results and limits our understanding of how dexmedetomidine might affect epidural or spinal anesthesia. The internal validity of this small randomized-controlled trial may have suffered at the expense of generalizability.

Finally, when interpreting safety outcomes such as bradycardia, a low incidence of adverse events in small datasets may dramatically underestimate the real risk when thousands of patients (rather than tens) are exposed to an intervention. ${ }^{20}$ Given the number of patients in each group, the zero incidence of bradycardia in each group has an upper $95 \%$ confidence interval of approximately $7.5 \%{ }^{20}$ Further study will be required to confirm that the incidence of bradycardia using this intervention in this population is safe.

Because of the above limitations, this study's impressive results should be interpreted with some caution. With possible nuances in the mechanism for shivering between labour epidural and spinal anesthesia, the groups were not mechanistically equivalent. Lamontagne $e t a l^{2}$ should be commended on their innovative study that provides an important contribution to the literature on a problem in obstetrical anesthesia still lacking an optimal solution. We hope this study will be a stimulus for further definitive research within mechanistically similar groups as we continue to strive towards improved patient-centred care in obstetrical anesthesia.

\section{La dexmédétomidine pour tous les accouchements par césarienne... ou peut-être pas?}

Les frissons périopératoires sont un problème clinique fréquemment observé et bien connu des anesthésiologistes qui prennent soin des femmes accouchant par césarienne. On estime que l'incidence médiane des frissons chez les parturientes recevant une analgésie péridurale ou une césarienne avec anesthésie péridurale ou rachianesthésie est de $52 \% .^{1}$ Les frissons sévères induisent un stress physiologique, interfèrent avec les moniteurs des patientes et réduisent le confort et la satisfaction globale des femmes pendant l'accouchement. ${ }^{2}$

Plusieurs études ont cherché à trouver des agents pharmacologiques afin de traiter les frissons associés à l'anesthésie neuraxiale, tant chez les parturientes que dans la population générale. Parmi les agents précédemment examinés, il a été démontré que le butorphanol, ${ }^{3}$ la clonidine ${ }^{4}$ le fentanyl, ${ }^{5}$ la mépéridine,${ }^{6}$ le tramadol ${ }^{7}$ et la dexmédétomidine ${ }^{8}$ réduisaient les frissons survenant après une anesthésie neuraxiale. Cependant, des effets secondaires tels que l'hypotension, les nausées et vomissements, ou encore une sédation excessive, limitent l'utilité de certains de ces agents chez certaines populations de patients.

La dexmédétomidine, un agoniste sélectif des récepteurs $\alpha-2$, a été introduite dans la pratique clinique aux ÉtatsUnis en 1999. Cet agent est approuvé par Santé Canada en tant que sédatif de courte durée chez le patient adulte sous ventilation mécanique à l'unité de soins intensifs et comme sédatif chez le patient non intubé avant et/ou pendant une intervention chirurgicale. Depuis, il a été approuvé et est 
couramment utilisé chez les patients pédiatriques et en cas de sédation consciente chez les patients non intubés. ${ }^{9}$ L'utilisation clinique de la dexmédétomidine s'est toutefois répandue vers d'autres usages hors indications. En anesthésie obstétricale, cet agent est utilisé dans le cadre d'une analgésie intraveineuse multimodale pour le travail et post-césarienne, ${ }^{10-13}$ en tant qu'adjuvant neuraxial dans l'espace péridural pour le travail, ${ }^{14}$ et par voie intrathécale pour la prévention des frissons pendant un accouchement par césarienne. ${ }^{15}$

Dans ce numéro du Journal, Lamontagne et coll. $^{2}$ présentent une utilisation novatrice de cet agent chez des parturientes pour traiter les frissons pendant un accouchement par césarienne. Les auteurs ont réalisé une étude mono-site, randomisée, à double insu et contrôlée par placebo dans deux groupes parallèles auprès de 80 parturientes en bonne santé subissant une césarienne sous anesthésie neuraxiale (rachianesthésie ou péridurale) qui ont manifesté des frissons importants après l'accouchement. Les participantes ont été randomisées à recevoir soit un bolus de $30 \mu \mathrm{g}$ de dexmédétomidine IV, ou un volume égal de solution saline. Les auteurs ont observé que, 15 minutes après l'administration, les frissons avaient complètement disparu chez $90 \%$ des patientes du groupe intervention vs seulement $23 \%$ des patientes du groupe témoin (risque relatif de ne pas frissonner, 4,0 ; intervalle de confiance $95 \%, 2,2$ à 7,2). Aucune bradycardie significative, un effet secondaire fréquemment remarqué lors de l'administration intraveineuse de dexmédétomidine, n'a été observée dans l'un ou autre groupe, et il n'y a eu qu'un cas d'hypotension chez une patiente du groupe intervention. Les auteurs ont donc conclu que les frissons apparaissant pendant un accouchement par césarienne pouvaient être traités de manière efficace et sécuritaire par la dexmédétomidine.

Cette étude propose une nouvelle option intrigante, séduisante même, afin de traiter un problème fréquemment rencontré dans la population obstétricale. Lorsqu'ils sont importants, les frissons constituent un désagrément pour l'anesthésiologiste qui s'occupe d'une patiente, et ils ont un impact sur l'expérience et la satisfaction des patientes concernant les soins que nous leur prodiguons. En se fondant sur cette étude, sommes-nous prêts à intégrer la dexmédétomidine dans notre pratique usuelle pour traiter les frissons chez les patientes obstétricales, étant donné qu'il s'agit d'une option hors indication (off-label) (et possiblement non rentable)? Avant de convaincre nos institutions d'autoriser l'utilisation de la dexmédétomidine dans une telle application, il convient d'examiner les limites de cette étude.

Lamontagne et coll. ${ }^{2}$ ont inclus des parturientes, en travail ou non, nécessitant une césarienne. La physiologie des parturientes change considérablement lorsqu'elles sont en travail. Il a été rapporté que les parturientes en travail pouvaient souffrir tant de frissons de régulation thermique que de frissons non régulateurs, avec ou sans analgésie péridurale. ${ }^{16}$ Malgré les augmentations normales de la température pendant le travail ${ }^{17}$ et avec l'administration d'une analgésie péridurale, ${ }^{18}$ des frissons peuvent tout de même survenir et leur mécanisme pourrait différer des frissons dus à la régulation de la température. ${ }^{1}$ En outre, les patientes en travail sont souvent exposées à une analgésie péridurale ainsi qu'à ses effets sur la régulation thermique et les frissons pendant plusieurs heures avant d'accoucher par césarienne. Ainsi, une parturiente en travail ayant reçu une analgésie péridurale et une parturiente dont le travail n'a pas commencé mais qui a reçu une rachianesthésie en vue d'une césarienne constituent deux populations de patientes vraisemblablement très distinctes.

En outre, en raison de leurs critères d'inclusion, chacun des groupes comptait certaines parturientes ayant reçu une rachianesthésie (70\%) et certaines ayant reçu une anesthésie péridurale $(30 \%)$ pour leur césarienne. Bien que la rachianesthésie et l'anesthésie péridurale réduisent toutes deux le seuil de frissonnement, nous ne savons pas si ces deux modalités affectent le seuil de frissonnement de la même façon ou différemment. ${ }^{1}$ Alors que la rachianesthésie a pour résultat un bloc dense situé au-dessous d'un certain dermatome, la péridurale épargne souvent les dermatomes sacrés, ce qui pourrait entraîner une réduction du bloc de l'afférence de l'apport thermique lors d'une anesthésie péridurale vs une rachianesthésie, indépendamment de la hauteur du bloc. ${ }^{1}$ La rachianesthésie agit rapidement pour induire une hypothermie et une température interne plus basse, alors qu'en cas d'anesthésie péridurale, les températures internes baissent lentement et de façon plus linéaire. ${ }^{19}$ Au delà de 30 minutes, les deux techniques font baisser la température interne à la même vitesse. ${ }^{19}$ Malgré des changements physiologiques généralement plus prononcés et une température interne plus basse, on a observé que les frissons accompagnant la rachianesthésie étaient moins intenses que ceux survenant avec une péridurale. ${ }^{19}$

Selon les auteurs, du fait qu'ils ont inclus une vaste population obstétricale, leur étude peut être davantage généralisable. Les auteurs ont tout de même réussi à obtenir des groupes intervention et témoin comparables en termes de travail actif et d'un nombre identique de patientes recevant une anesthésie péridurale $v s$ rachidienne et ce, même sans stratifier ces facteurs. Cette question de sous-groupes ayant des pronostics possiblement différents ou additifs entraîne des résultats incertains et limite notre compréhension de la façon dont la dexmédétomidine pourrait affecter différemment l'anesthésie péridurale ou rachidienne. La validité interne 
de cette petite étude randomisée contrôlée pourrait avoir souffert de son désir d'être généralisable.

Enfin, lors de l'interprétation de critères d'innocuité tels que la bradycardie, une faible incidence d'événements indésirables dans de petits ensembles de données pourrait radicalement sous-estimer le risque réel lorsque des milliers de patientes (au lieu de dizaines) sont exposées à une intervention. ${ }^{20}$ Étant donné le nombre de patientes de chaque groupe, l'incidence zéro de bradycardie dans chaque groupe donne une limite supérieure de l'intervalle de confiance de $95 \%$ à environ 7,5\%. ${ }^{20}$ Des études supplémentaires seront nécessaires afin de confirmer que l'incidence de bradycardie lors de l'utilisation de cette intervention dans cette population est véritablement sans risque.

En raison des écueils mentionnés ci-dessus, les résultats impressionnants de cette étude devraient être interprétés avec prudence. D'un point de vue mécaniste, les groupes n'étaient pas équivalents, étant donné les différences possibles dans le mécanisme sous-jacent aux frissons provoqués par une péridurale ou une rachianesthésie. Lamontagne et coll. ${ }^{2}$ doivent être félicités pour leur étude novatrice qui constitue une contribution majeure à la littérature concernant un problème touchant l'anesthésie obstétricale et pour lequel nous ne disposons pas encore de solution optimale. Nous espérons que cette étude motivera d'autres recherches approfondies dans des groupes semblables d'un point de vue mécaniste; en attendant de trouver une solution définitive à ce problème, nous maintiendrons nos efforts pour améliorer les soins aux patientes en anesthésie obstétricale.

Conflicts of interest None declared.

Editorial responsibility This submission was handled by Dr. Philip M. Jones, Associate Editor, Canadian Journal of Anesthesia.

\section{Conflit d'intérêt Aucun.}

Responsabilité éditoriale Cet article a été traité par Dr Philip M. Jones, rédacteur adjoint, Journal canadien d'anesthésie.

\section{References}

1. Crowley LJ, Buggy DJ. Shivering and neuraxial anesthesia. Reg Anesth Pain Med 2008; 33: 241-52.

2. Lamontagne C, Lesage S, Villeneuve É, Lidzborski E, Derstenfeld $A$, Crochetière $C$. Intravenous dexmedetomidine for the treatment of shivering during cesarean delivery under neuraxial anesthesia: a randomized-controlled trial. Can J Anesth 2019; 66. DOI: https://doi.org/10.1007/s12630-019-01354-3

3. Juneja M, Ackerman WE 3rd, Heine MF, Cases-Cristobal V, Urella RP, Rigor BM. Butorphanol for the relief of shivering associated with extradural anesthesia in parturients. J Clin Anesth 1992; 4: 390-3.

4. Mercadante S, De Michele P, Letterio G, Pignataro A, Sapio M, Villari $P$. Effect of clonidine on postpartum shivering after epidural analgesia: a randomized, controlled, double-blind study. J Pain Symptom Manage 1994; 9: 294-7.

5. Matthews NC, Corser G. Epidural fentanyl for shaking in obstetrics. Anaesthesia 1988; 43: 783-5.

6. Casey WF, Smith CE, Katz JM, O'Loughlin K, Weeks SK. Intravenous meperidine for control of shivering during caesarean section under epidural anaesthesia. Can J Anaesth 1988; 35: 12833.

7. Mahesh T, Kaparti L. A randomised trial comparing efficacy, onset and duration of action of pethidine and tramadol in abolition of shivering in the intra operative period. J Clin Diagn Res 2014; 8: GC07-9.

8. Abdel-Ghaffar HS, Mohamed SA, Fares KM, Osman MA. Safety and efficacy of dexmedetomidine in treating post spinal anesthesia shivering: a randomized clinically controlled dosefinding trial. Pain Physician 2016; 19: 243-53.

9. Jun JH, Kim KN, Kim JY, Song SM. The effects of intranasal dexmedetomidine in children: a systemic review and metaanalysis. Can J Anesth 2017; 64: 947-61.

10. Elterman KG, Meserve JR, Wadleigh M, Farber MK, Tsen LC. Management of labor analgesia in a patient with acute myeloid leukemia. A A Case Rep 2014; 3: 104-6.

11. Nie $Y, T u W$, Shen $X$, et al. Dexmedetomidine added to sufentanil patient-controlled intravenous analgesia relieves the postoperative pain after cesarean delivery: a prospective randomized controlled multicenter study. Sci Rep 2018; 8: 9952.

12. Mazy A, Gad M, Bedairy M. Preperitoneal postcesarean section bupivacaine analgesia: comparison between dexamethasone and dexmedetomidine as adjuvants. Saudi J Anaesth 2018; 12: 183-9.

13. Yousef AA, Salem HA, Moustafa MZ. Effect of mini-dose epidural dexmedetomidine in elective cesarean section using combined spinal-epidural anesthesia: a randomized doubleblinded controlled study. J Anesth 2015; 29: 708-14.

14. Zhang W, Li C. ED50 of epidural ropivicaine combined with dexmedetomidine for labor analgesia. Clin J Pain 2018; 34: 9503.

15. Mio S, Shi M, Zou L, Wang G. Effect of intrathecal dexmedetomidine on preventing shivering in cesarean section after spinal anesthesia: a meta-analysis and trial sequential analysis. Drug Des Devel Ther 2018; 12: 3775-83.

16. Panzer O, Ghazanfari N, Sessler DI, et al. Shivering and shivering-like tremor during labor with and without epidural analgesia. Anesthesiology 1999; 90: 1609-16.

17. Marx GF, Loew DA. Tympanic temperature during labour and parturition. Br J Anaesth 1975; 47: 600-2.

18. Fusi L, Steer PJ, Maresh MJ, Beard RW. Maternal pyrexia associated with the use of epidural analgesia in labour. Lancet 1989; 1: 1250-2.

19. Saito T, Sessler DI, Fugita K, Ooi Y, Jeffrey R. Thermoregulatory effects of spinal and epidural anesthesia during cesarean delivery. Reg Anesth Pain Med 1998; 23: 418-23.

20. Hanley JA, Lippman-Hand A. If nothing goes wrong, is everything all right?Interpreting zero numerators. JAMA 1983; 249: 1743-5.

Publisher's Note Springer Nature remains neutral with regard to jurisdictional claims in published maps and institutional affiliations. 